\title{
Lymphoepithelial cyst in the pancreatic accessory spleen:A case report
}

\author{
Sawako Hiroi ${ }^{1}$, Michinori Hamaoka ${ }^{2}$, Rie Yamamoto ${ }^{1}$, Yasuhiro Matsugu², Takashi \\ Nishisaka $^{1}$, Hideki Nakahara ${ }^{2}$, and Toshiyuki Itamoto ${ }^{2}$ \\ ${ }^{1}$ Affiliation not available \\ ${ }^{2}$ Hiroshima Prefectural Hospital
}

January 25, 2021

\begin{abstract}
Lymphoepithelial cyst (LEC)is a rare disease. Clear diagnostic criteria have not been established, and the number of cases is expected to increase in the future. In particular, LEC in the pancreatic accessory spleen has not been reported in the past, and this report documents it for the first time.
\end{abstract}

\section{Introduction}

Pancreatic cysts are broadly categorized as neoplastic intraductal papillary mucinous neoplasm (IPMN), mucinous cystic neoplasm (MCN), serous cystic neoplasm (SCN), and non-neoplastic (pancreatic pseudocysts, lymphoepithelial cysts, epidermoid cysts, and dermoid cysts). In addition, there are pancreatic neuroendocrine tumors (pNET), in which the tumor has degenerated into cysts. International clinical practice guidelines for pancreatic cystic tumors include the International Association of Pancreatology (IAP) guidelines, ${ }^{1}$ European expert consensus statements, ${ }^{2}$ and the American Gastroenterological Association (AGA) guidelines. ${ }^{3}$ However, there are no guidelines for pancreatic pseudocysts and no clear diagnostic criteria have been established. Pancreatic lymphoepithelial cysts (LECs) are rare benign lesions and were initially described by Luchtrath and Schriefers in $1985 .{ }^{4}$ In 1987, Truong proposed to call it a lymphoepithelial cyst. ${ }^{5}$ The cysts are typically observed in middle-aged and elderly men; they occur equally in the pancreatic head, body, or tail, and may present as a single or multilocular lesion. This study presents the case of a patient with a LEC in the pancreatic accessory spleen. This is the first such instance being documented. We believe that our report is valuable as it is the first of its kind on LEC in the pancreatic accessory spleen. Furthermore, the exposure and knowledge acquired through it will enable the development of international diagnostic criteria for LECs and similar epidermoid cysts and dermoid cysts in the future.

\section{Key Clinical Message}

Lymphoepithelial cysts (LECs) have not been documented previously. This report is the first of its kind and given that the number of cases of LECs is expected to increase in the future, this report sets the precedent for future research.

\section{Presentation of case}

A 43-year-old male patient was found to have a pancreatic tail cyst upon abdominal ultrasonography on physical examination. He had no subjective symptoms, or medical history. Laboratory data, such as complete blood cell count, hepatic and renal functions, as well as serum levels of carcinoembryonic antigen (CEA) and carbohydrate antigen 19-9 (CA 19-9) were within conventional limits. In addition, amylase, lipase, and blood glucose levels were normal. On further referral to the study hospital, computed tomography (CT) 
revealed a cyst with a diameter of $15 \mathrm{~mm}$ in the pancreatic tail. The cystic lesion was monocystic and had an intensity-enhanced area around the cyst in the low intensity area (Fig. 1). Magnetic resonance imaging (MRI) revealed a lesion with depleted and enhanced intensities on the T1 and T2-weighted figures, respectively. Diffusion-weighted MRI displayed increased signal potency in the peripheral portion of the cystic lesion (the wall and the septa); the cystic contents presented reduced intensity on an enhanced MRI (Fig. 2).

Endoscopic ultrasonography (EUS) revealed a cyst with high-echoic lesions, a pancreatic tail, and calcification on the cyst margin (Fig. 3a). Endoscopic retrograde cholangiopancreatography (ERCP) displayed a slight displacement of the pressure superior to the duct, with no narrowing or disruption (Fig. 3b).

The findings of suspected cyst degeneration on CT and MRI with low intensity on T1-weighted figures and high intensity on T2-weighted figures were consistent with the characteristics of pNET. The preoperative diagnosis was pNET. According to the guidelines mentioned above, the first-line of treatment for pNET is surgery. A distal pancreatectomy was performed with concomitant splenectomy. The postoperative course was uneventful. The excised surface of the resected specimen revealed a multilocular cyst with solid nodules (Fig. 4a). Histopathological findings revealed the spleen, as well as, the red and white pulp in the parenchyma of the pancreatic tail. Since spleen tissue was found in the pancreatic tissue, we diagnosed it as an intrapancreatic accessory spleen cyst. The major and minor multilocular cysts had a maximum diameter of $17 \mathrm{~mm}$; the luminal epithelium consisted of mature squamous epithelium with display of the subepithelial lymphoid tissue. The cyst lumen revealed keratin and cholesterol deposits in the clefts. The fact that lymphatic tissue occupies the majority of the cyst is a major feature of LECs and meets the diagnostic criteria for LECs. Ultimately, the patient was diagnosed with a LEC of the pancreatic accessory spleen.

\section{Discussion}

The accessory spleen is not uncommon. Halpert et al. reported 291 (10.8\%) of 2700 autopsied cases with an accessory spleen, $215(62.1 \%)$ in the vicinity of the splenic hilum, followed by $78(22.5 \%)$ in the pancreatic tail $^{6}$. However, a cyst occurring in the pancreatic accessory spleen is extremely rare (Table 1). ${ }^{7-52}$ The lesion site was the pancreatic tail in all cases. The primary complaints were abdominal pain and vomiting. On the other hand, 33 of 57 cases were asymptomatic. Smaller tumor diameters are often asymptomatic. The case reported in our study has a tumor diameter of $1.5 \mathrm{~cm}$, which is the smallest among the reported cases, and it is estimated that there were no symptoms. CEA or CA 19-9 was elevated in some cases, but remained within the conventional limits in others. However, the lesion was asymptomatic in many cases, and detected by image examination. Cyst morphology was multilocular in 33 cases, and unilocular in 15. The tumor diameter ranged from $15 \mathrm{~mm}$ to $134 \mathrm{~mm}$. A majority of the findings were reduced in T1, and elevated in T2-weighted MRI images. Preoperative diagnoses, such as IPMN, pNET, epidermoid cyst, and malignant tumor suggest case complexity. Consequently, presurgical diagnosis of pNET was performed. Histopathological findings revealed LEC in the present case; no such instance has been reported previously.

Pancreatic LECs are extremely rare. LECs are reported to account for $0.5 \%$ of pancreatic cysts; they were initially identified and reported by Luchtrath et al. in $1985^{4}$, and so named by Truong et al. in $1987 . .^{5}$ Mege et al. reported a study with pancreatic LECs in middle-aged to elderly men (mean age, 55 years; range, 20-82 years; 91 men vs. 26 women); it was observed that the lesion was occasionally accompanied by abdominal pain (43\%) and an elevated serum CA 19-9 level (55\%). ${ }^{53}$ Pancreatic cysts can be classified into true cysts, pseudocysts, and cystic neoplasms. LEC is considered as a type of true cyst characterized by a lining of squamous epithelium with dense subepithelial lymphoid tissue. The cystic contents are typically white in color and may include keratinized material or cholesterol crystals. Adsay et al. classified cystic lesions covered by the squamous epithelium of the pancreas as LECs; those occurring in the sub-pancreatic epithelium are epidermoid and dermoid cysts with cutaneous appendages. ${ }^{54}$ The pathological diagnostic criteria for LECs are ambiguous. Presently, the predominant diagnostic criteria of a LEC comprises the lumen surface of the cyst with stratified squamous epithelium with abundant lymphoid tissue underneath. The reports so far follow the classification of cysts as defined by Adsay et al. ${ }^{54}$ Adsay et al. defined that "LECs are characterized microscopically by stratified squamous epithelium surrounded by a band of mature lymphoid 
tissue with intervening well-formed germinal centers". In addition to the above, Adsay et al. defined "The second type of squamous-lined cyst in the pancreas is the epidermoid cyst arising in intrapancreatic accessory spllen". Adsay et al. do not mention lymphoid-rich cysts in the accessory spleen. Among the cases reported as epidermoid cysts around 1987 proposed by Truong, there were some cases that were thought to be LECs. ${ }^{5}$ Due to the unclear classification, it cannot be ruled out that LECs may also be included in the cases reported as epidermoid cysts.

In the present case, the lumen epithelium of the multilocular cyst consisted of mature squamous material with developed sub-epithelial lymphoid tissue. In addition, the white and the red pulp were detected in the pancreatic accessory spleen and diagnosed as splenic LEC. No such LECs have been reported; consequently, the present case is extremely rare. There are multiple theories regarding the pathogenic mechanism of pancreatic LECs. The first theory suggests an origin from the misplaced branchial cleft tissue because of the histologic resemblance. The second suggests squamous metaplasia on an obstructed pancreatic duct, which subsequently protrudes into a peripancreatic lymph node. The third indicates cyst development from an ectopic pancreas in a peripancreatic lymph node. At present, a decisive view has not been reached. Tateyama et al. reported, "In the present immunohistochemical study, the cytokeratin phenotypes of the epithelial lining of LEC were similar to those of the epithelial retention cysts but different from those of branchial cleft cysts. ${ }^{14}$ In addition to the cytokeratin pattern, the presence of some islets and ducts in the fibrous wall of the LEC might support the second hypothesis". In this case, a LEC was found in the accessory spleen in the pancreas, and we reckon that that the second hypothesis that squamous metaplasia on the pancreatic duct protrudes into the pancreatic lymph tissue of the accessory spleen in the pancreas is a highly plausible explanation for our case. However, it still needs consideration and discussion.

\section{Conclusion}

This report outlines the first instance of a LEC in the intrapancreatic accessory spleen. The diagnostic criteria for LECs are ambiguous, and the difference from epidermoid cysts is unclear. It is necessary to consider LECs from a pathological point of view.

\section{Author Contributions}

SH drafted the manuscript. MH and TI supervised the preparation of the manuscript. YM contributed to the surgery. RY and TN contributed to the pathological diagnosis. MH, YM, RY, TN, and TI reviewed and modified the manuscript. The authors read and approved the final manuscript.

\section{Acknowledgements}

We would like to thank Editage (www.editage.com) for English language editing.

\section{Conflict of Interest statement}

The authors declare that they have no competing interests.

Funding The authors received no funding for this study.

\section{Figure Legends}

Fig. 1: Computed tomography (CT): A cystic mass with a diameter of $15 \mathrm{~mm}$ in the pancreatic tail (arrow).

Fig. 2: Magnetic Resonance Imaging (MRI): A. Cystic lesion measuring $18 \times 14 \mathrm{~mm}$ cystic lesion assessed by low signal intensity on T1-weighted image(arrow). B. The same lesion with high signal intensity on T2-weighted image (arrow).

Fig. 3: Endoscopic imaging: A. Endoscopic ultrasonography (EUS) showed a cyst with calcification in the margin (arrow). B. Endoscopic retrograde cholangiopancreatogtaphic image showing a normal main pancreatic duct with no communication with the cystic lesion.

Fig. 4: Histopathology: A. The cystic wall is lined by mature keratinized squamous epithelium and underlying lymphoid tissue. B. The epithelial lining is surrounded by splenic pulp and pancreatic tissue. 


\section{Tables}

Table.1: English literature on cysts in the accessory spleen in the pancreas

\section{References}

1. Tanaka, M., Fernández-Del Castillo, C., Kamisawa, T., Jang, J. Y., Levy, P., Ohtsuka, T., Salvia, R., Shimizu, Y., Tada, M., Wolfgang, C. L. 2017. Revisions of international consensus Fukuoka guidelines for the management of IPMN of the pancreas. Pancreatology 17:738-753.

2. European evidence-based guidelines on pancreatic cystic neoplasms. Gut 2018, 67 (5), 789-804.

3. Vege, S. S., Ziring, B., Jain, R., Moayyedi, P. 2015. American gastroenterological association institute guideline on the diagnosis and management of asymptomatic neoplastic pancreatic cysts. Gastroenterology 148:819-22.

4. Lüchtrath, H., Schriefers, K. H. 1985. A pancreatic cyst with features of a so-called branchiogenic cyst. Pathologe 6:217-219.

5. Truong, L. D., Rangdaeng, S., Jordan, P. H., Jr. 1987. Lymphoepithelial cyst of the pancreas. American J Surg Pathol 11:899-903.

6. Halpert, B., and Alden, Z. A. 1964. Accessory spleens in or at the tail of the pancrease. A survery of 2,700 addiotnal necropsies. Archives of Pathology 77:652-654.

7. Davidson, E. D., Campbell, W. G., Hersh, T. 1980. Epidermoid splenic cyst occurring in an intrapancreatic accessory spleen. Digestive Diseases and Sciences 25:964-967.

8. Hanada, M., Kimura, M., Kitada, M., Nakajima, T., Yamada, K., Yoshii, M. 1981. Epidermoid cyst of accessory spleen. Acta Patholologica Japanica 31:863-872.

9. Morohoshi, T., Hamamoto, T., Kunimura, T., Yoshida, E., Kanda, M., Funo, K., Nagayama, T., Maeda, M., Araki, S. 1991. Epidermoid cyst derived from an accessory spleen in the pancreas. A case report with literature survey. Acta Pathologica Japanica 41:916-921.

10. Nakae, Y., Hayakawa, T., Kondo, T., Shibata, T., Kitagawa, M., Sakai, Y., Sobajima, H., Ishiguro, H., Tanikawa, M., Nimura, Y. 1991. Epidermoid cyst occurring in a pancreatic accessory spleen. Journal of Clinical Gastroenterology 13:362-364.

11. Tang, X., Tanaka, Y., Tsutsumi, Y. 1994. Epithelial inclusion cysts in an intrapancreatic accessory spleen. Pathology International 44:652-654.

12. Furukawa, H., Kosuge, T., Kanai, Y., Mukai, K. 1998. Epidermoid cyst in an intrapancreatic accessory spleen: CT and pathologic findings. American Journal of Roentgenology 171:271.

13. Higaki, K., Jimi, A., Watanabe, J., Kusaba, A., Kojiro, M. 1998. Epidermoid cyst of the spleen with CA199 or carcinoembryonic antigen productions: Report of three cases. American Journal of Surgical Pathology 22:704-708.

14. Tateyama, H., Tada, T., Murase, T., Fujitake, S., Eimoto, T. 1998. Lymphoepithelial cyst and epidermoid cyst of the accessory spleen in the pancreas. Modern Pathology 11:1171-1177.

15. Sasou, S., Nakamura, S., Inomata, M. 1999. Epithelial splenic cysts in an intrapancreatic accessory spleen and spleen. Pathology International 49:1078-1083.

16. Choi, S. K., Ahn, S. I., Hong, K. C., Kim, S. J., Kim, T. S., Woo, Z. H., Shin, S. H. 2000. A case of epidermoid cyst of the intrapancreatic accessory spleen. Journal of Korean Medical Science 15:589-592.

17. Tsutsumi, S., Kojima, T., Fukai, Y., Kanoh, K., Shimura, T., Mochiki, E., Kato, R., Asao, T., Kuwano, H. 2000. Epidermoid cyst of an intrapancreatic accessory spleen-A case report. Hepatogastroenterology 47:1462-1464. 
18. Horibe, Y., Murakami, M., Yamao, K., Imaeda, Y., Tashiro, K., Kasahara, M. 2001. Epithelial inclusion cyst (epidermoid cyst) formation with epithelioid cell granuloma in an intrapancreatic accessory spleen. Pathology International 51:50-54.

19. Sonomura, T., Kataoka, S., Chikugo, T., Hirooka, T., Makimoto, S., Nakamoto, T., Sato, M. 2002. Epidermoid cyst originating from an intrapancreatic accessory spleen. Abdominal Imaging 27:560-562.

20. Fink, A. M., Kulkarni, S., Crowley, P., Crameri, J. A. 2002. Epidermoid cyst in a pancreatic accessory spleen mimicking an infected abdominal cyst in a child. American Journa of Roentgenology 179:206-208.

21. Yokomizo, H., Hifumi, M., Yamane, T., Hirata, T., Terakura, H., Murata, K., Fujita, H., Matsukane, H. 2002. Epidermoid cyst of an accessory spleen at the pancreatic tail: Diagnostic value of MRI. Abdominal Imaging 27:557-559.

22. Kanazawa, H., Kamiya, J., Nagino, M., Uesaka, K., Yuasa, N., Oda, K., Arai, T., Nishio, H., Nimura, Y. 2004. Epidermoid cyst in an intrapancreatic accessory spleen: A case report. Journal of Hepatobiliary Pancreatic Surgery 11:61-63.

23. Ru, K., Kalra, A., Ucci, A. 2007. Epidermoid cyst of intrapancreatic accessory spleen. Digestive Diseases and Sciences 52:1229-1232.

24. Itano, O., Shiraga, N., Kouta, E., Iri, H., Tanaka, K., Hattori, H., Suzuki, F., Otaka, H. 2008. Epidermoid cyst originating from an intrapancreatic accessory spleen. Journa of Hepatobiliary and Pancreatic Surgery 15:436-439.

25. Servais, E. L., Sarkaria, I. S., Solomon, G. J., Gumpeni, P., Lieberman, M. D. 2008. Giant epidermoid cyst within an intrapancreatic accessory spleen mimicking a cystic neoplasm of the pancreas: Case report and review of the literature. Pancreas 36:98-100.

26. Gleeson, F. C., Kendrick, M. L., Chari, S. T., Zhang, L., Levy, M. J. 2008. Epidermoid accessory splenic cyst masquerading as a pancreatic mucinous cystic neoplasm. Endoscopy 40:141-142.

27. Zhang, Z., and Wang, J. C. 2009. An epithelial splenic cyst in an intrapancreatic accessory spleen. A case report. Journal of the Pancreas 10:664-666.

28. Reiss, G., Sickel, J. Z., See-Tho, K., Ramrakhiani, S. 2009. Intrapancreatic splenic cyst mimicking pancreatic cystic neoplasm diagnosed by EUS-FNA. Gastrointestinal Endoscopy 70:557-558.

29. Kadota, K., Kushida, Y., Miyai, Y., Katsuki, N., Hayashi, T., Bando, K., Shibuya, S., Haba, R. 2010. Epidermoid cyst in an intrapancreatic accessory spleen: Three case reports and review of the literatures. Pathology \& Oncology Research 16:435-442.

30. Panagiotopoulos, N., Acharya, M., Ahmad, R., Bansi, D., Jiao, L. 2012. Epithelial inclusion cyst arising within an intra-pancreatic splenunculus. International Journal of Surgery Case Reports 3:118-120.

31. Horn, A. J., and Lele, S. M. 2011. Epidermoid cyst occurring within an intrapancreatic accessory spleen. A case report and review of the literature. Journa of the Pancreas 12:279-282.

32. Iwasaki, Y., Tagaya, N., Nakagawa, A., Kita, J., Imura, J., Fujimori, T., Kubota, K. 2011. Laparoscopic resection of epidermoid cyst arising from an intrapancreatic accessory spleen: A case report with a review of the literature. Surgical Laparoscopy Endoscopy \& Percutaneous Techiques 21:275-279.

33. Yamanishi, H., Kumagi, T., Yokota, T., Koizumi, M., Azemoto, N., Watanabe, J., Mizuno, Y., Sugita, A., Abe, M., Ikeda, Y., Matsuura, B., Hiasa, Y., Onji, M. 2011. Epithelial cyst arising in an intrapancreatic accessory spleen: A diagnostic dilemma. Internal Medicine 50:1947-1952.

34. Urakami, A., Yoshida, K., Hirabayashi, Y., Kubota, H., Yamashita, K., Hirai, T., Tsunoda, T. 2011. Laparoscopy-assisted spleen-preserving pancreatic resection for epidermoid cyst in an intrapancreatic accessory spleen. Asian Journal of Endoscopic Surgery 4:185-188. 
35. Khashab, M. A., Canto, M. I., Singh, V. K., Hruban, R. H., Makary, M. A., Giday, S. 2011. Endosonographic and elastographic features of a rare epidermoid cyst of an intrapancreatic accessory spleen. Endoscopy 43:193-194.

36. Harris, A. C., Chaudry, M. A., Menzies, D., Conn, P. C. 2012. Laparoscopic resection of an epidermoid cyst within an intrapancreatic accessory spleen: A case report and review article. Surgical Laparoscopy Endoscopy \& Percutanenou Techniques 22:246-249.

37. Hong, R., Choi, N., Sun, K., Lim, S., Han, Y. 2013. Epidermoid cyst arising from an intrapancreatic accessory spleen: A case report and review of the literature. Oncology Letters 5:469-472.

38. Hamidian Jahromi, A., Fallahzadeh, M. K., Dela Cruz, N., Chu, Q. 2013. Epidermoid cyst arising from an intrapancreatic accessory spleen: A case report and a review of the literature. Journal of the Louisiana State Medical Society 165:153-156.

39. Zavras, N., Machairas, N., Foukas, P., Lazaris, A., Patapis, P., Machairas, A. 2014. Epidermoid cyst of an intrapancreatic accessory spleen: A case report and literature review. World Journal of Surgical Oncology $12: 92$.

40. Kumamoto, Y., Kaizu, T., Tajima, H., Kubo, H., Nishiyama, R., Watanabe, M. 2015. A rapidly growing epidermoid cyst in an intrapancreatic accessory spleen treated by laparoscopic spleen-preserving distal pancreatectomy: Report of a case. International Journal of Surgery.

41. Kwak, M. K., Lee, N. K., Kim, S., Han, G. J., Seo, H. I., Park, D. Y., Lee, S. J., Kim, T. U. 2016. A case of epidermoid cyst in an intrapancreatic accessory spleen mimicking pancreas neoplasms: MRI with DWI. Clinical Imaging 40:164-166.

42. Kato, S., Mori, H., Zakimi, M., Yamada, K., Chinen, K., Arashiro, M., Shinoura, S., Kikuchi, K., Murakami, T., Kunishima, F. 2016. Epidermoid cyst in an intrapancreatic accessory spleen: Case report and literature review of the preoperative imaging findings. Internal Medicine 55:3445-3452.

43. Modi, R. M., Kamboj, A. K., Swanson, B., Conwell, D. L., Krishna, S. G. 2016. Epidermoid cyst within an intrapancreatic accessory spleen: Endosonography and confocal endomicroscopy of an unusual pancreatic cystic lesion. Endoscopy 48:332-333.

44. Fujii, M., Yoshioka, M., Shiode, J. 2016. Two cases of an epidermoid cyst developing in an intrapancreatic accessory spleen identified during laparoscopic distal pancreatectomy. Internal Medicine 55:3137-3141.

45. Hirabayashi, K., Yamada, M., Kono, H., Hadano, A., Kawanishi, A., Takanashi, Y., Kawaguchi, Y., Nakagohri, T., Mine, T., Nakamura, N. 2017. Epidermoid cysts are a characteristic feature of intrapancreatic but not of extrapancreatic accessory spleens. Virchows Archiv: European Journal of Pathology 471:91-98.

46. Matsumoto, K., Kato, H., Okada, H. 2018. Epidermoid cyst in an intrapancreatic accessory spleen diagnosed by typical radiographic images and endoscopic ultrasound fine-needle aspiration findings with contrast agent. Clinical Gastroenterolog and Hepatology 16:13-14.

47. Takagi, C., Hoshi, N., Kikuchi, Y., Shirakawa, H., Tomikawa, M., Ozawa, I., Hishinuma, S., Ogata, Y. 2020. Epidermoid cyst within an intrapancreatic accessory spleen exhibiting abrupt changes in serum carbohydrate antigen 19-9 level: A case report. Surgical Case Reports 6:133.

48. Shan, G. D., Chen, W. G., Hu, F. L., Chen, L. H., Yu, J. H., Zhu, H. T., Gao, Q. Q., Xu, G. Q. 2017. A spontaneous hematoma arising within an intrapancreatic accessory spleen: A case report and literature review. Medicine 96:8092.

49. Paredes, A., Beal, E. W., Dillhoff, M. E. 2018. Epidermoid cyst within an intrapancreatic accessory spleen. BMJ Case Reports.

50. Zhou, B., Zhang, Q., Zhan, C., Ding, Y., Yan, S. 2018. Laparoscopic spleen-preserving pancreatic resection for epidermoid cyst in an intrapancreatic accessory spleen: Case report and literature review. Therapeutics 
and Clinical Risk Managemen 14:937-944.

51. Watanabe, H., Yamaguchi, Y., Ohtsubo, K., Mouri, H., Motoo, Y., Yamashita, K., Minamoto, T., Gabata, T., Sawabu, N. 2004. Epidermoid cyst of the intrapancreatic accessory spleen producing CA19-9. Digestive Endoscopy 16:244-248.

52. Won, J.K., Lee, Y. J., Kang, G.H. 2005. Epithelial cyst in the intrapancreatic accessory spleen that clinically mimic pancreatic cystic tumor. The Korean Journal of Pathology 39:437-441.

53. Mege, D., Grégoire, E., Barbier, L., Del Grande, J., Le Treut, Y. P. 2014. Lymphoepithelial cyst of the pancreas: An analysis of 117 patients. Pancreas 43:987-995.

54. Adsay, N. V., Hasteh, F., Cheng, J. D., Klimstra, D. S. 2000. Squamous-lined cysts of the pancreas: Lymphoepithelial cysts, dermoid cysts (teratomas), and accessory-splenic epidermoid cysts. Seminars in Diagnostic Pathology 17:56-65.

\section{Hosted file}

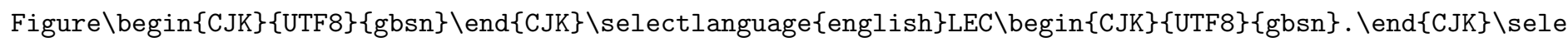
available at https://authorea.com/users/391527/articles/505603-lymphoepithelial-cyst-in-thepancreatic-accessory-spleen-a-case-report

\section{Hosted file}

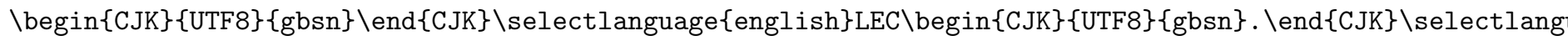
available at https://authorea.com/users/391527/articles/505603-lymphoepithelial-cyst-in-thepancreatic-accessory-spleen-a-case-report

\section{Hosted file}

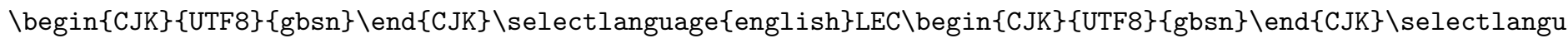
available at https://authorea.com/users/391527/articles/505603-lymphoepithelial-cyst-in-thepancreatic-accessory-spleen-a-case-report 